\title{
Cervical ectopic pregnancy, conservative management, report of two cases and review of the bibliography
}

\begin{abstract}
Ectopic pregnancy represents around $1 \%$ of all pregnancies, it is considered an entity of difficult conservative management, in patients who wish to preserve fertility, in the context of hospitals, which cannot offer approaches with minimal invasion such as hysteroscopy, without However, conservative treatment with cervical balloon placement, clamping of the uterine arteries with the ZEA technique, and doses of methotrotexate have been documented. Cases with meted handling.
\end{abstract}

Keywords: cervical ectopic pregnancy, report case, ZEA technique uterine artery clamping, endocervical balloon, conservative management
Volume II Issue 4 - 2020

\author{
Yanet Fermin-Aldama, 'Cesar Rodriguez- \\ Villan, ${ }^{2}$ Fernando Martinez Gonzalez ${ }^{3}$ \\ 'Resident of gynecology and obstetrics, Department of Naval \\ Medical Center, Mexico \\ ${ }^{2}$ Affiliated Physician, Naval Medical Center, México \\ ${ }^{3}$ Private medicine, Mexico
}

\begin{abstract}
Correspondence: Dr.Yanet fermin Aldama, Resident physician of gynecology and obstetrics, naval medical center, Coyoacán delegation, zip code 04480. Mexico City, Tel +52 962I525285, Email yanet_aldama@hotmail.com
\end{abstract}

Received: June 15, 2020 | Published: July 202020

\section{Introduction}

Extrauterine pregnancy is defined when the pregnancy is not located at the endocavitary level. ${ }^{1}$ Therefore, ectopic pregnancy, where implantation occurs in the cervical canal, ${ }^{2}$ makes diagnosis and treatment difficult for young women who do not yet have satisfied motherhood, therefore, strategies are sought to avoid hysterectomy as usual treatment. ${ }^{2}$

The incidence has increased in the last 30years, and although its morbidity and mortality has decreased, it is still considered the first cause of bleeding in the first half of pregnancy with high mortality in women.

Cervical ectopic pregnancy is considered to have a frequency of less than $1 \%$ of all ectopic pregnancies, with an incidence of one case every 1-000-95,000 pregnancies. $^{2}$

Patients with a history of ectopic pregnancy have an affected prognosis for subsequent fertility. Its complications reduce the chances of an intrauterine pregnancy occurring ( $40 \%$ of cases treated with salpingectomy). ${ }^{3}$

\section{Regarding its location}
a) Ampullar $80 \%$
b) $12 \%$ isthmic
c) Fimbric 5\%
d) Interstitial $2 \%$
e) abdominal- $1 \%$

Extra tubal pregnancy: $1.7 \%$
a. Ovarian pregnancy: intrafollicular or interstitial $0.15 \%$
b. Abdominal pregnancy: primary or secondary $1.4 \%$
c. Cervical pregnancy $0.15 \%$

The etiology is unknown. Among the risk factors that have been described in the literature are all those processes (mainly iatrogenic) that cause damage to the endometrial line or the cervix, such as cervical dilation and curettage, Asherman's syndrome, anterior caesarean section, uterine or cervical surgery, and intrauterine devices. ${ }^{4}$

The increase in cervical pregnancy rates in recent years is closely related to the increasingly widespread use of in vitro fertilization techniques with embryo implantation. ${ }^{1}$

Its early diagnosis is an important factor in the vital and reproductive prognosis of patients with such a complication. ${ }^{2}$

The diagnosis of suspected ectopic pregnancy is established, until proven otherwise, in every sexually active woman of childbearing age who consults for irregular bleeding and pain in the lower abdomen, when the levels of $\beta-\mathrm{HCG}$ are $>6500 \mathrm{mU} / \mathrm{ml}$ and an intrauterine gestational sac is not observed, the diagnosis of ectopic is very likely. ${ }^{5}$ However, false positive images are observed in $20 \%$ of ectopics within the uterus. It can also be observed, the existence of liquid in the bottom of the Douglas sac, whose presence may be secondary, among others, to the ruptured ectopic. ${ }^{2}$ It can be affirmed that there is suspicion of Ectopic if in a woman who has levels of $\beta-\mathrm{HCG}>3000 \mathrm{mU} /$ $\mathrm{ml}$ transvaginal ultrasound does not detect an intrauterine gestational sac. $^{3}$

If the diagnosis is established before bleeding occurs, trophoblastic invasion can be reduced with conservative medical treatments, such as the administration of methotrexate. 3,6

\section{Clinical case presentation}

22-year-old patient, 2 caesarean section 1, with no relevant history for the current condition, went to the emergency department with a pregnancy of 7.5 weeks of gestation due to the date of last menstruation and long-standing transvaginal bleeding, denies abdominal pain, on physical examination he found vital signs within normal parameters, with no evidence of peritoneal irritation, on gynecological examination with transvaginal bleeding. 
The endovaginal ultrasound reported a uterine cavity with a $14 \mathrm{~mm}$ endometrial mid echo, bilaminar and homogeneous. Cervix with ovoid image of echogenic guresus wall, anechoic interior, gestational sac with image inside with a $4 \mathrm{~mm}$ embryo, in relation to $6 \mathrm{sdg}$ pregnancy (Figure 1) (Figure 2). With beta fraction of $9145 \mathrm{mUI} / \mathrm{mL}$. Ectopic pregnancy is diagnosed.

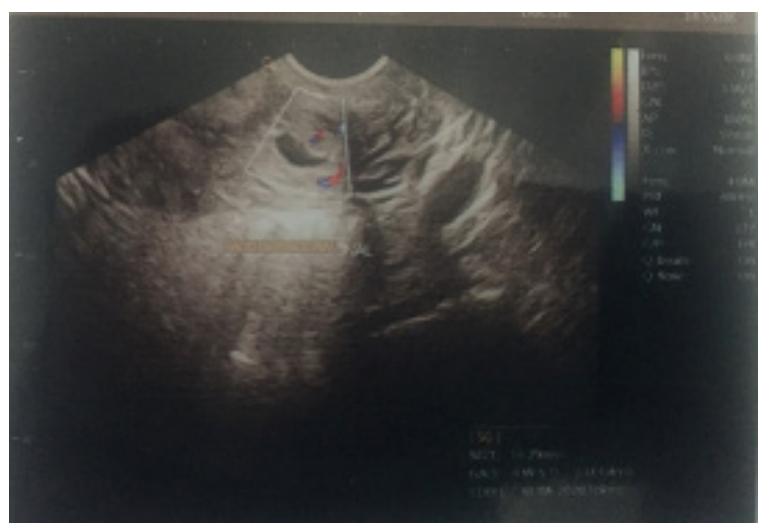

Figure I cervix with gestational sac.

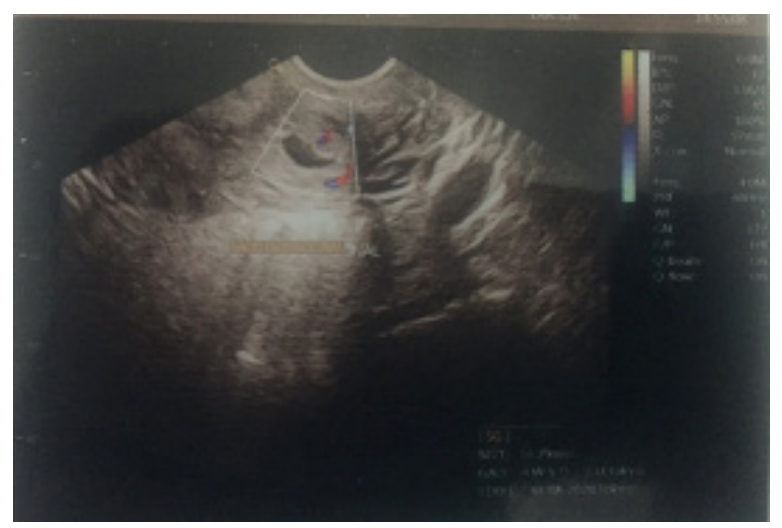

Figure 2 Cervix doppler application gestational sac.

The patient expressed a desire for fertility, conservative management was decided, surgical management was started consisting of manual uterine aspiration, clamping of the uterine arteries with the zea technique, placement of an intracervical foley catheter with $30 \mathrm{cc}$ of solution, to prevent bleeding from the surgical bed, in postoperatively, management is started with methotrexate at $50 \mathrm{mg}$ or single dose.

With adequate postoperative evolution, the foley catheter was removed 24 hours after surgery, and was discharged on the third day, without complications. With transvaginal control ultrasound with a report of the uterine cavity with a 3-mm endometrial mid-echo, cervix ultrasound normal, ovaries without alterations.

\section{Case number 2}

38-year-old patient, 42, 2 caesarean section 2, with no relevant history for the current condition, fum. 08.01.2020 comes for presenting transvaginal bleeding with an 8 -week pregnancy by date of last menstruation, the physical examination found vital signs within normal parameters, no data on peritoneal irritation, gynecological examination with transvaginal bleeding.

Endovaginal ultrasound reported a uterine cavity with a $10-\mathrm{mm}$ endometrial, bilaminar and homogeneous echo. Cervix with ovoid image, anechoic interior, gestational sac with image inside with $9.7 \mathrm{~mm}$ embryo, in relation to $8 \mathrm{sdg}$ pregnancy, with fetal heart rate (Figure 3).

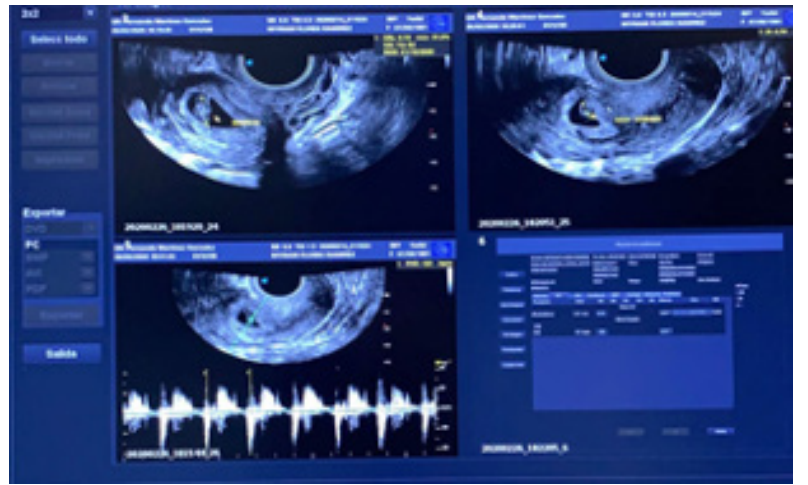

Figure 3 Cervix with ovoid image, anechoic interior, gestational sac with image inside with $9.7 \mathrm{~mm}$ embryo.

Surgical treatment was decided, conservative management was decided, surgical management consisting of manual uterine aspiration was started, clamping of the uterine arteries with the zea technique, placement of an intracervical foley catheter with $30 \mathrm{cc}$ of solution, to prevent surgical bed bleeding, however identifies obstetric hemorrhage, which does not refer to conservative management, for which reason obstetric hysterectomy is performed (Figure 4).

With histopathological report, post gestational uterus, with cervical implantation, from the first trimester of gestation, and abnormally adherent partial placentation, hemorrhage at the implantation site (Figure 4).

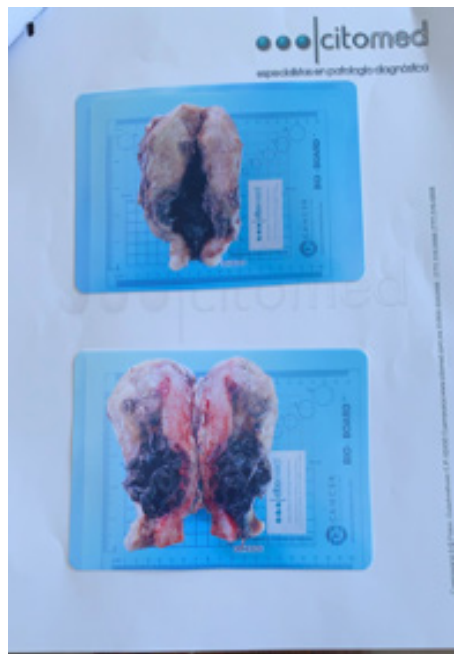

Figure 4 Post gestational uterus, with cervical implantation, first trimester of gestation.

\section{Discussion}

Given the low incidence of cervical ectopic pregnancy, conservative schemes for tubal ectopic pregnancy are found in the current literature, and few case reports are similar to ours. It is important to mention that there are no randomized clinical studies on conservative treatment. However, given the history of our patient, the same was decided.

Cervical ectopic pregnancy treatment options can be divided into procedures that: 
i. They allow a hemostatic tamponade.

ii. They reduce the contribution of blood flow to the ectopic.

iii. They split the trophoblastic tissue.

iv. They act as feticides (intraamniotic or systemic route).

Regarding tamponade procedures, the most widespread is the use of a foley catheter, which must have a $30 \mathrm{ml}$ balloon that can be inflated to $100 \mathrm{ml}$. Haemostasis rates have been published in around $90 \%$ of the cases in which it is applied.

Techniques that reduce blood flow to pregnancy are adjuncts to other procedures, as a preparation or as a rescue method for profuse bleeding. There are several methods: cerclage, vaginal ligation of the cervical arteries, ligation of the internal or uterine iliac artery, local vasoconstrictors such as vasopressin, and embolizations.

Among the techniques that divide the trophoblast there are 2 classic procedures: hysterectomy and curettage. Primary hysterectomy may be appropriate in cases of uncontrollable bleeding, diagnoses in the second trimester of pregnancy, and in situations in which you want to avoid blood transfusions or emergency surgery, especially if there is no desire to preserve fertility. Curettage is often combined with other mechanical techniques, such as cervical artery ligation and balloon tamponade. Even so, it has been reported that $20 \%$ of them end up with a hysterectomy. Finally, regarding the use of feticides, there are local and systemic treatment options.

Substances such as etoposide and actinomycin D have been used in systemic chemotherapy, but the most commonly used is methotrexate, which is more effective the earlier the gestational age, although successes have been reported even in pregnancies with visible heartbeat and $\beta$-HCG values $>40,000$ IU. There are single-dose and multiple-dose regimens, with and without folinic rescue, but the data is too limited to compare the efficacy of the different regimens with each other.

Some authors have reported the use of other substances, such as ethanol and lipiodol. Methotrexate was first used in 1983. Applications have been described at different doses and routes (systemic, intraamniotic and cervical). Furthermore, treatment guidelines have been reported in combination with other procedures, such as curettage and tamponade. In general, it is usually effective in cases of 12 weeks or less; Success rates have been published, in terms of uterine preservation of $91 \%$. The proportion of cases requiring concomitant surgical procedure in cases of viable cervical ectopic pregnancy is $43 \%$. Success rates of $81.3 \%$ have been described in early diagnoses treated with chemotherapy. ${ }^{7}$

Conservative management has a better prognosis than surgery and is an option even in twin stations (heterotopic or not). Therefore, surgery should be the last resort, when conservative treatment has failed. ${ }^{3,8}$ This is because patients treated with surgery are more exposed to hemorrhage and hysterectomy. The use of methotrexate alone or in combination with other procedures has not detracted from the reproductive capacity of patients in whom the uterus may be preserved. On the other hand and considering that most pregnancies after a cervical ectopic pregnancy end at term, it should be noted that, although the half-life of methotrexate is $10 \mathrm{~h}$, it can remain in the liver and maternal kidney for months. For this reason, and in order to avoid possible teratogenic risks, some authors recommend a 6-month wait before trying to achieve a new pregnancy. 6,7
The diagnosis of cervical ectopic pregnancy is difficult, it does not always meet the ultrasound criteria, so it can go unnoticed and lead to inappropriate treatments that produce serious complications, such as a hysterectomy in a patient with a desire for pregnancy. Treatments aimed at avoiding hysterectomy are complicated and not without risk, currently treatment is offered by hysteroscopy, with total resolution, however not all hospitals have this procedure, the appropriate treatment continues to be the performance of a hysterectomy.

\section{Conclusion}

Since there are no randomized clinical trials, the prognosis of patients undergoing conservative treatments is unknown; the different chemotherapy regimens have not been established for cervical ectopic pregnancy.

Appropriate surgical treatments for these patients are unknown in terms of therapeutic improvement; if it is better AMEU, LUI, Cervical tamponade, Impingement of uterine arteries with the ZEA technique or ligation of hypogastric arteries with the Gala technique. For our patient in particular, they were applied in a staggered manner, appropriately restraining the bleeding, each device being removed in the same way.

In the case presented, adequate evolution and conservation of fertility. Follow-up will be necessary in the next pregnancies to assess the prognosis of this patient.

\section{Acknowledgments}

None.

\section{Funding}

None.

\section{Conflicts of interest}

All authors declare that they have no competing interests.

\section{References}

1. Centre Center for fetal and neonatal medicine of barcelona, tubal and non-tubal ectopic pregnancy. Hospital clinic, University of Barcelona; 2018.

2. Parodi MR, Monteiro A, Di Trápani N, et al. Gynecological imaging and interventional work: diagnosis and treatment of cervical ectopic pregnancy. ). Revista de Imagenología. 2017;20(2):60-64.

3. Amilcar BR, José AF, Mildret MA. Cervical ectopic pregnancy. Hysteroscopic treatment, presentation of a case. Ginecol Obstet Mex. 2015;83(5):302-307.

4. Pozo Cevallos, Gloria Celeste. Accident ectopic pregnancy in a 26-yearold patient. Tesis de Licenciatura; 2018.

5. Mouhajer M, Samuel OBED, Amalachukwu MO. Cervical ectopic pregnancy in resource deprived areas: A rare and difficult diagnosis. Ghana medical journal. 2017;51(2):94-97.

6. Bolaños-Bravo HH, Ricaurte-Fajardo A, Zarama-Márquez F, et al. A Conservative management in a patient with cervical ectopic pregnancy in Nariño, Colombia: case report and literature review. Colombian Journal of Obstetrics and Gynecology. 2019;70(4):282-297.

7. Elósegui Jesús, Joaquín Hijona. Conservative approach to cervical ectopic pregnancy. Obstetrics and gynecology progress. 2008;51(1):4146. 